\title{
METAMODELING ACTIVITIES FOR HIERARCHICAL COMPONENT-BASED MODELS
}

\author{
Abdurrahman Alshareef \\ Hessam Sarjoughian \\ Arizona Center for Integrative $M \& S$ \\ Schl. of Comp., Info., \& Deci. Syst. Engr. \\ Arizona State University \\ 699 South Mill Avenue \\ Tempe, AZ, 85281, USA \\ \{alshareef,sarjoughian\}@asu.edu
}

\begin{abstract}
Abstractions are necessary to facilitate grounding and managing model development and simulation experiments. Understanding the specific roles of different artifacts in abstractions is important to enrich the model development process. Actions and states reveal certain unique aspects about system behavior within and among component models. However, behavior specifications are not explicit and adequately supported in state-based formalisms. In this paper, we examine the role of actions in model specification in accordance with the notion of state in the modular and hierarchical parallel DEVS formalism. The resulting approach relies on the profoundness of DEVS while having a more intuitive abstraction around it via hierarchical activity metamodeling. Together the activity and DEVS modeling help simplify and strengthen specifying complex system behaviors as composition of atomic and coupled models. We show the importance of hierarchical DEVS activity modeling approach with a coordinator model as a part of a dual-server model.
\end{abstract}

Keywords: Abstraction, Action, Activity, Behavior, Specification.

\section{INTRODUCTION}

Models can be perceived in different ways especially at a meta-layer. It is important to examine different models and metamodels as representations of some component. However, issues may arise due to using different representations and possibly at different layers. Model-Driven Architecture (MDA) has been proposed (Soley et al. 2000) to provide a framework to work with abstract and concrete models across a four-layer architecture. It defines a semi-formal approach to guide the process of developing models and metamodels across these layers and the relationships between different layers and within the same layer. Essential relationships are instantiation, interpretation, conformance, and transformation.

Metamodeling is used to provide a means to describe systems in general (Henderson-Sellers 2012) and relationship between systems of systems. A key to metamodeling is to identify and relate essential artifacts of a system of interest from higher level abstractions. The problem is evident since these abstractions are representatives of their corresponding realizations to some lower level abstraction and eventually in the implementation space. Multiple understandings are invoked when metamodeling certain elements of the system. As such, handling differences becomes quite challenging especially when system complexity is 
high or partially known. Representing instances raises issues in metamodeling too. According to (OMG 2017), the notion of instantiation is only meaningful within metamodel. And the relationship across metalayers is defined through interpretations of the model from the higher layer in the immediate lower layer.

The model itself is one of the four basic entities in the modeling and simulation (M\&S) framework (Zeigler, Muzy, and Kofman 2018). It forms the basic component with a sound mathematical foundation. The model is conceived as a set of instructions by a simulator. Therefore, it simulates the model and correctly produces its state and output trajectories based on the input trajectories for a given initial state. According to the I/O requirement for deductive, deterministic models (Wymore 1993), one unique output trajectory is generated for each input trajectory and initial state. As such, the definition of the model has a precise semantics which is not the case in the existing semi-formal methods such as the MDA and UML (Unified Modeling Language) (OMG 2012).

Actions and states are two commonly used concepts when ascribing system behavior. They provide suitable means for abstractions about behavioral system specifications. However, their semantics may pose challenges when they are considered at some high layer in the MDA hierarchy. A key aspect of the problem resides in having a way for interpreting states and actions into concrete system models. The relation between action and state can be collectively examined as a basis for providing a means by which their definitions and specifications are used together to describe system dynamics. The relation can be examined from individual as well as integrative standpoints. In both ways, complementary perspective of their roles is taken to account for richer behavioral specifications where neither is known to be sufficient to produce proper manifestations at the implementation level.

This work is focused around the trio of component, state, and action, in forming abstractions about complex systems. We examine relations between action and state at a higher level from one side, and their possible corresponding representation in the theory of modeling and simulation on the other side. We also discuss the relation between the two abstractions themselves, the action and the state. The discussion is grounded in the context of metamodeling activities where the role of action is collectively played with control toward behavioral specifications of the component-based models.

In the following section (Section 2), we discuss the notion of component modeling in the context of this work. In Section 3, we describe the server systems as perceived in the system-theoretic literature. In Section 4, we discuss behavioral specifications across the different layers for metamodeling activities. We discuss the related work and the concluding remarks in the Sections 5 and 6, respectively.

\section{COMPONENT-BASED MODELING}

As much as we are concerned about identifying components with respect to their structure, it is also important to understand their behavior by which system dynamics are defined. Components at the higher level can bear hierarchical relationships with other components. An example is the template model (Zeigler and Sarjoughian 2017). The template model can be extended by some instance template model. Instance template models can be transformed into instance models thereafter. The relationships between the aforementioned model types allows modelers to incrementally develop models while obtaining simulations after fully providing their specifications.

Specialized components define a type of relationship where some components share and/or mimic structures and behaviors of some other components. Therefore, a component specializes another such that it redefines its structure and behavior (i.e., as properties, functions, and relationships are added to a component, the component becomes a more accurate representation of its counterpart in the real system). After that, the instance model type transforms the instance template model and choose all specialization relationships. The resulting model can have multiple components via decomposition. 


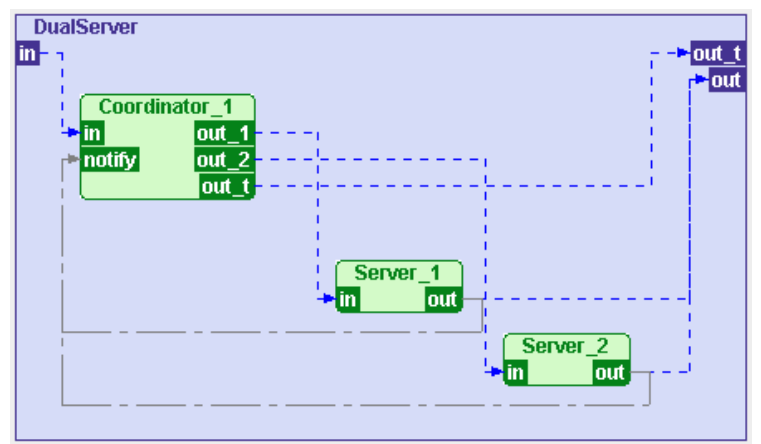

(a) The components, ports and couplings for the dual server model

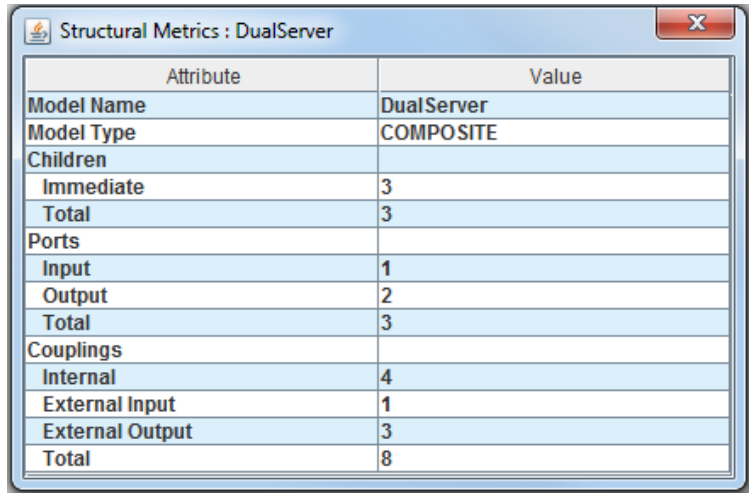

(b) The structural metrics for the dual server model

Figure 1: Modeling the dual server system in CoSMoS.

In Figure 1, a hierarchical component view is designed using the Component-based System Modeling and Simulation (CoSMoS) (ACIMS 2017). The tool's framework provides a means by which components can be defined along with their couplings whether they are internal, external input, or external output coupling based on the system being modeled. Both primitive and composite models can be defined as components with possibly composition and specialization relationships. Considering the Discrete Event System Specification (DEVS) formalism, primitive and composite models correspond to atomic and coupled models, respectively. Atomic model functions are the state transition functions including the internal $\left(\delta_{\text {int }}\right)$ and the external $\left(\delta_{\text {ext }}\right)$ transition function, the output function $(\lambda)$, and the time advance function $(t a)$. The confluence function $\left(\delta_{c o n}\right)$ is defined to further exploit parallelism in the Parallel DEVS formalism. In CoSMoS, such behavior can be defined using a variant of statecharts devised to incur certain aspects of the behavioral specifications in a state-based manner (Fard and Sarjoughian 2015). Coupled model is defined as a composite component which has a finite number of components thereof along with input and output ports and couplings. An external input coupling defines the coupling between an input port of a coupled model and an input port of one of its components. An external output coupling defines the coupling between an output port of a contained component and an output port of a coupled component. An internal coupling is defined between two components of a coupled model. The DEVS formalism is modular such that communication between components are only allowed through coupling and their designated ports. Component receives input or bag of inputs in arbitrary manner and thereafter it may only accept them according to the correspondent specifications.

The dual server composite template model is devised to include three components, one instance of the coordinator template model, and two instances of the server template model. The couplings are defined as in Figure 1a. The components are identified to mimic dual server system such that job is assigned to the first server if it is available, otherwise, it gets assigned to the second server. If both servers are busy, then the job is added to a queue in order to wait for one of the servers to become available. The dual server coupled model is defined with one input port for receiving jobs. It is also defined with two output ports. One port is sending notifications about the job being dispatched to its corresponding server, and another is for producing the completed jobs. The coordinator template is defined with two input ports and three output ports. An input port is for receiving jobs, and another port is designated for receiving notification when the job gets completed which means that the corresponding server becomes available. In addition, the coordinator is defined with three output ports. Two of them are for sending output to servers. The third one is coupled with the dual server out $_{t}$ port which is defined for experimental purposes. The server template is defined with one input port for receiving assigned jobs and one output port for producing them after they are serviced. 
An internal coupling is defined for each server output to the notify input port of the coordinator to notify it about the completion of its job and as a consequence the availability of the corresponding server.

The formal specification of the dual server coupled model is the following

$$
A=\left\langle X, Y, D,\left\{M_{d} \mid d \in D\right\}, E I C, E O C, I C\right\rangle, \text { where }
$$

InPorts $=\{$ in $\}$, where $\quad X_{\text {in }}=V$ (an arbitrary set $), \quad X_{M}=\{(v) \mid v \in V\}$

OutPorts $=\left\{\right.$ out $\left._{\text {out }}\right\}$, where $X_{p}=V, \quad Y_{M}=\{(p, v) \mid v \in V$ and $p \in$ OutPorts $\}$

$D=\left\{\right.$ coordinator $_{1}$, Server $_{1}$, Server $\left._{2}\right\}$

$M_{\text {coordinator }}=$ Coordinator $; M_{\text {Server }_{1}, \text { Server }_{2}}=$ Server

EIC $=\{(($ DualServer, in $),($ coordinator, in $))\}$

EOC $=\left\{\left(\left(\right.\right.\right.$ coordinator $_{1}$, out $\left._{t}\right),\left(\right.$ DualServer out $\left.\left._{t}\right)\right),\left(\left(\right.\right.$ Server $_{1}$, out $),($ DualServer, out $\left.)\right)$,

$\left(\left(\right.\right.$ Server $_{2}$, out $),($ DualServer, out $\left.\left.)\right)\right\}$

$I C=\left\{\left(\left(\right.\right.\right.$ coordinator $_{1}$, out $\left._{1}\right),\left(\right.$ Server $_{1}$, in $\left.)\right),\left(\left(\right.\right.$ coordinator $_{1}$, out $\left._{2}\right),\left(\right.$ Server $_{2}$, in $\left.)\right)$,

$\left(\left(\right.\right.$ Server $_{1}$, out $),\left(\right.$ coordinator $_{1}$, notify $\left.)\right),\left(\left(\right.\right.$ Server $_{2}$, out $),\left(\right.$ coordinator $_{1}$, notify $\left.\left.)\right)\right\}$

Some aspects of the complexity of this model is determined by looking into the components and the communication between them. Figure $1 \mathrm{~b}$ shows the structural metrics after defining the model in CoSMoS. The metrics of components, ports and couplings, are easy to compute since all elements of the dual server persist in a relational database. Different coordination mechanisms could result in different measures. Moreover, different designs of the same coordination mechanism could also result in different measures. The ability to easily observe such metrics becomes important especially when such models become larger and ordinarily more complex. Models of such nature are developed to grow especially when accounting for such complexity at the early stage of design. Further examination of such metrics is necessary to understand their meaning, if they have one, whether in domain specific settings or in general.

The ability to be characterized with scale and complexity traits (Sarjoughian 2017) is important even though measures for behavioral traits maybe taken qualitatively. Quantitative measures are usually taken for structural aspects such as the ones in Figure 1b. In the context of metamodeling activities, some metrics about syntactical properties of actions and flows whether they are control or data could be easily obtained. More information can be also obtained about the input/output pins and control nodes. However, these information needs to be further examined to determine their relevance in contributing to the overall behavioral scale and complexity traits.

\section{COORDINATING BETWEEN SERVER COMPONENTS}

The server system (Wymore 1993) consists of servers along with a queuing component that coordinates dispatching of jobs to one or more server components. The server system can accept inputs via three ports. The first port is for receiving elements of the queue. The second port is to accept the service time produced by the generator according to some probability distribution such as the exponential distribution. The third port is added to receive a random Boolean to indicate the existence of an error to determine the result of conducting the processing operation. In the model shown in Figure 1a, we omit this port, and instead we designate a port for the receipt of the job completion by a server. The job itself and the service time are both accepted via the input port in.

State variables for Parallel DEVS atomic models are categorized into primary and secondary variable types. The primary state variables are generally defined by phase and sigma $(\sigma)$. In the coordinator component, they are used to determine how the model responds to the arrival of a job whether by storing it in a queue or 
sending it out to the corresponding server. In the case where some server is available, the sigma is assigned to zero time advance and therefore sends out the job to the selected available server. Otherwise, if there is no available server, the job is stored in a FIFO queue.

In addition, the state of the coordinating unit includes several secondary variables. The received job is maintained along with its accepted service time in advance to assigning it to some designated server. The coordinator includes a queue to hold on the received jobs in the case that all servers are busy. The stored jobs in the queue are also time stamped by their arrival time in order to facilitate analyzing their turnaround time at a later stage. Another state variable of the coordinator is an array to maintain the availability status of each server. When a server is assigned a job, its availability status is changed to be false until receiving the job completion notification from it. It is changed to true afterward. The determined server $i d$ for assigning the job is also maintained in order to send out the job through its designated port and coupling. Hence, the number of servers is static and therefore known by the coordinator during the initialization stage. The current model does not account for structural changes during the simulation.

An activity model corresponding to the external transition function of the coordinator is depicted in Figure 2. This model is devised using our previous activity-based DEVS model specification approach (Alshareef, Sarjoughian, and Zarrin 2016). It describes coordination and assignment of jobs to servers (see 1a). Inputs are received and then processed in an iterative manner by the expansion region. For each job arriving through the in port, the coordinator model either assigns it to an available server or stores it in its queue. In the case of assigning a job to a server, the sigma set to zero and the phase set to sending for immediate dispatching of the jobs. For the inputs arriving through the notify port, the availability of each server is updated since each received input indicates processing of a job has been completed and therefore its assigned server becomes available. When a server is available and there are jobs waiting to be processed, then as many jobs as possible are removed from the queue and dispatched to available servers and marking them as unavailable. This type of activity is characterized with fine-grain nodes with details about the inner specification of the atomic model. The activities in the Figures 3 and 5 are collectively specified and therefore reside at different abstraction layers.

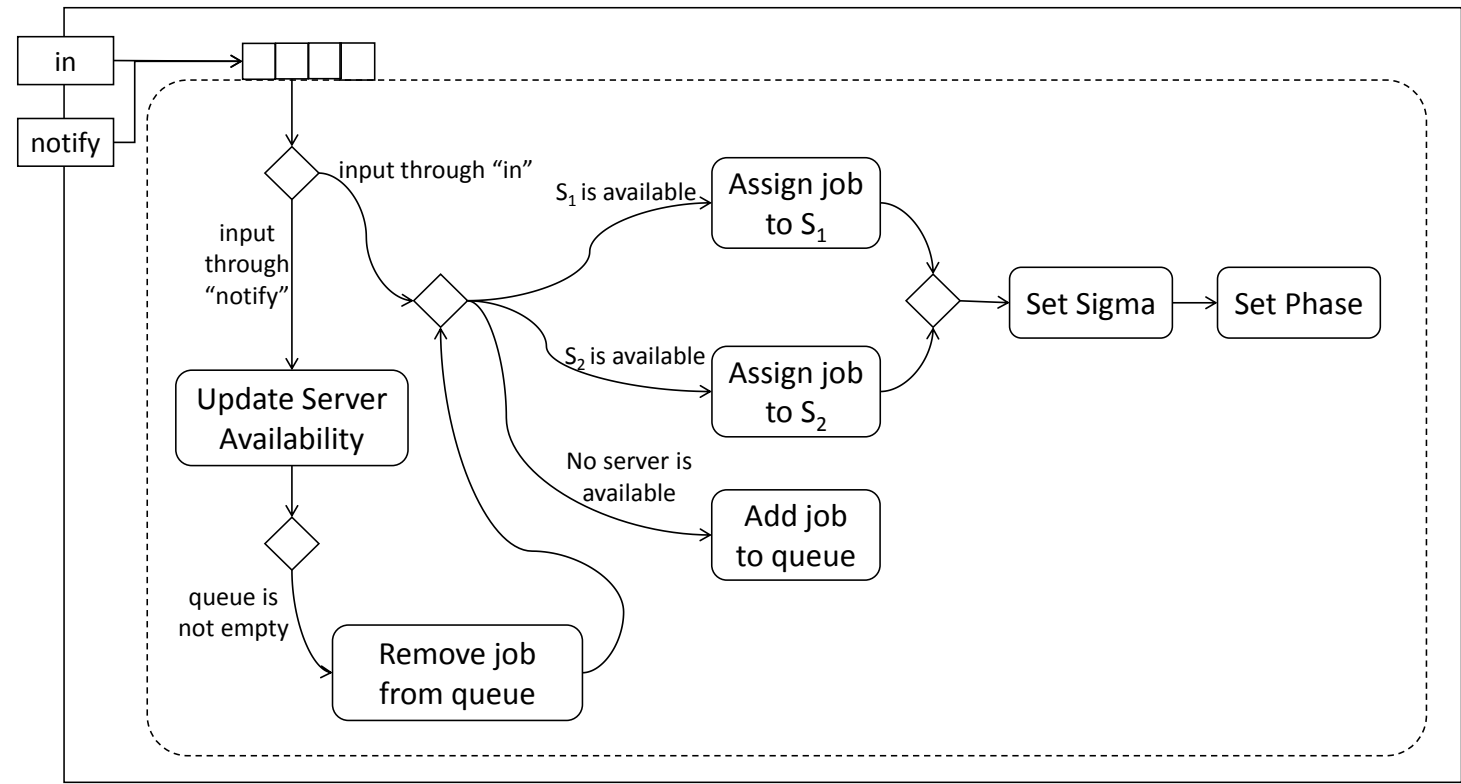

Figure 2: An activity for the external transition function of the coordinator. 
The coordinating unit can be viewed as a component or multiple components of the multi-processing architecture system. Different coordination can be also considered to account for different processing architectures such as the pipeline and divide \& conquer (Alshareef and Sarjoughian 2018). Changes on coordination can be achieved through state definitions or manipulation of the primary or secondary state variables. They can be also achieved via different designs of the components and their couplings. The coordinator can be considered as a subsystem within a larger subsystem or the system itself. Such subsystem can consist of one component or multiple components where they are all closed under coupling in order to collectively achieve the coordinating task. In the context of activities, parts of the coordinating task are delegated to a set of control constructs to arrive at the intermediary stage of modeling different kind of processing architecture. For example, decision and split nodes can be used to enrich models at higher level for logic descriptions of the coordination task for multiple processor. On the one hand, Conditions of the outgoing flows from the decision node are employed to check for the availability of servers. On the other hand, dispatching outputs is synchronized for different servers such as in divide \& conquer or for the server and the transducer such as in the multi-server architecture. This synchronizing of such task is achieved through the split node.

Inputs to such system are generated externally and fed into it through the external input coupling. The flow is synchronized mainly at the arrival of inputs to the input port of the coordinator and at the end of completing the job after service. Therefore, servers can operate independently yet their outputs have to be synchronized. Such synchronization is depicted in activities by the merge and join nodes (the activity in Figure 3 is to be discussed in Section 4). Due to modularity, this dependency is exclusive. The components are stimulated only through their I/O. In Parallel DEVS, parallelism is exploited when possible by providing a means by which all imminent components can execute concurrently in any given simulation cycle.

Concurrent flows with single or multiple servers among them can provide a concrete basis to a wide range of semantics for control as well as data flows among the activities. Useful simulations can be obtained to observe and monitor different behaviors of models that have been attained incrementally through complementary views of their representations at higher layers. The granularity of time base may although vary depending on the system as well as the input/output requirement. For example, the behavior can be observed for a limited time base for some purposes among which testing or debugging for specific scenarios can take place. Evaluation of the performance is also important and can be achieved through crafted simulations with specific observations capabilities.

\section{THE SPECIFICATION OF ACTION AND CONTROL IN ACTIVITIES}

The activities metamodel (OMG 2012) essentially consists of the major flow and node elements. The flow can be classified into control or data flow types. The node can be classified further in many different types. Action is a major node in activities. It is a fundamental unit in dictating the behavior of systems. Along with other types of node, such as control nodes, they form together the overall behavior in an encompassing activity. There are four major types of control nodes, decision, merge, split and join nodes. Each one has some semantics to handle flows in activities.

Action is an abstract classifier to represent a wide range of possibilities for model to behave in many different ways. In (OMG 2017), several types are introduced to provide a means to handle different behavioral aspects. For example, structural feature actions manipulate structural features including reading and writing. We conceive the notion of action based on system-theoretic principles, particularly the DEVS formalism. Therefore, it needs to align with the notion of component as well as state as defined in DEVS. Action is not merely an intermediary means upon state transitions. In (Shoham 1989), action defines state. In the context of activities, action and control elements are both specializations of the node type. They can be used to represent different dynamics at a higher level before realizing their implementations. In previous 
work (Alshareef and Sarjoughian 2018), we devised an interpretation facility to support the simulation of activities in DEVS-Suite simulator (ACIMS 2019).

The control nodes provides a suitable means to direct the flow including the ones carrying data. The coordination task can be specified using different variations of controlling constructs based on dynamics of the system of interest. For example, coordinating for an ordinary multi-server system requires directing jobs to different servers. It could also require maintaining the status of each server. The coordinator could also maintain the queue. The state variables of such system are discussed in Section 3. The controlling constructs of activities can be selected to be a consistent representation of the coordinator when possible considering the semantics of each construct. The decision node is used to determine which outgoing flow is selected. The merge node could receive multiple flows. As soon as it receives a flow, it directs it to the corresponding node. The split node is used to produce multiple flows concurrently while the join is used to synchronize multiple incoming flows. These constructs, and different combinations of them, can collectively serve as a representation for the desired coordination dynamics. These are significant relative to specifying behavior solely in terms of states and transitions with strong simplifications on actions.

Actions of activities can be equipped with input and output pins. Unlike actions, control nodes do not have such means. Therefore, as a semi-formal approach, it needs to be compensated with such definition of port as a necessary mechanism for handling the I/O. In the DEVS formalism, communication among different component can be only achieved through the designated ports. In our approach, action is encapsulated within component and yet explicitly specified to define the total behavior of the corresponding models. The multiplicity may vary based upon the complexity of the behavior of which the activity constructs are defined to represent (see Figure 1a). The activity itself can be equipped with input and output parameters. Both parameter and pin are specializations of the object node. They both serve as means for communications among and between activities. The former is for communicating between activities, and the latter is for communicating among them.

Thus, we create an activity of the multi-server archetype architecture (Figure 3). The activity receives its input through input parameter. The coordination decision node then decides based on state variables and depicted in the conditions $C_{1}$ and $C_{2}$ to which server the job is to be directed. The subsequent splitting nodes then produce two flows, one to the server, and another for Merge $e_{1}$ node. The flow to Merge ${ }_{1}$ is devised to

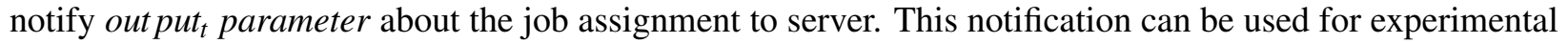
purposes such as performance evaluation. After servicing the job, a flow is issued from the server to Merge $_{2}$ which is then directed to the split node. The latter node produces two flows, one to output parameter, and another to notify the coordinating procedure about the availability status of the corresponding server.

For richer model specification, the semantics of the described connections in this approach are complemented by the semantics of couplings in Figure 1a and the semantics of flows in Figure 3. on the one hand, the flows are endowed with the formal and precise semantics of coupling. On the other hand, different types of flow at different layers provide the means for richer specification with the notions of control and data. Having such a capability is significant with proper use of model-driven methodologies across abstraction layers. The elements within the dashed line area in Figure 3 show the presence of two internal and one external output couplings with respect to the coordinator component.

\subsection{Coordinator statecharts}

The coordinator is devised as a component in Figure 1 and therefore its behavior can be looked into from statecharts standpoint. The statecharts (see Figure 4) mainly consists of two states, passive and sending. The passive state is to represent state of the coordinator when it is not manipulating any job. When receiving an input, then the coordinator checks through which input port the input has been received. Input through 


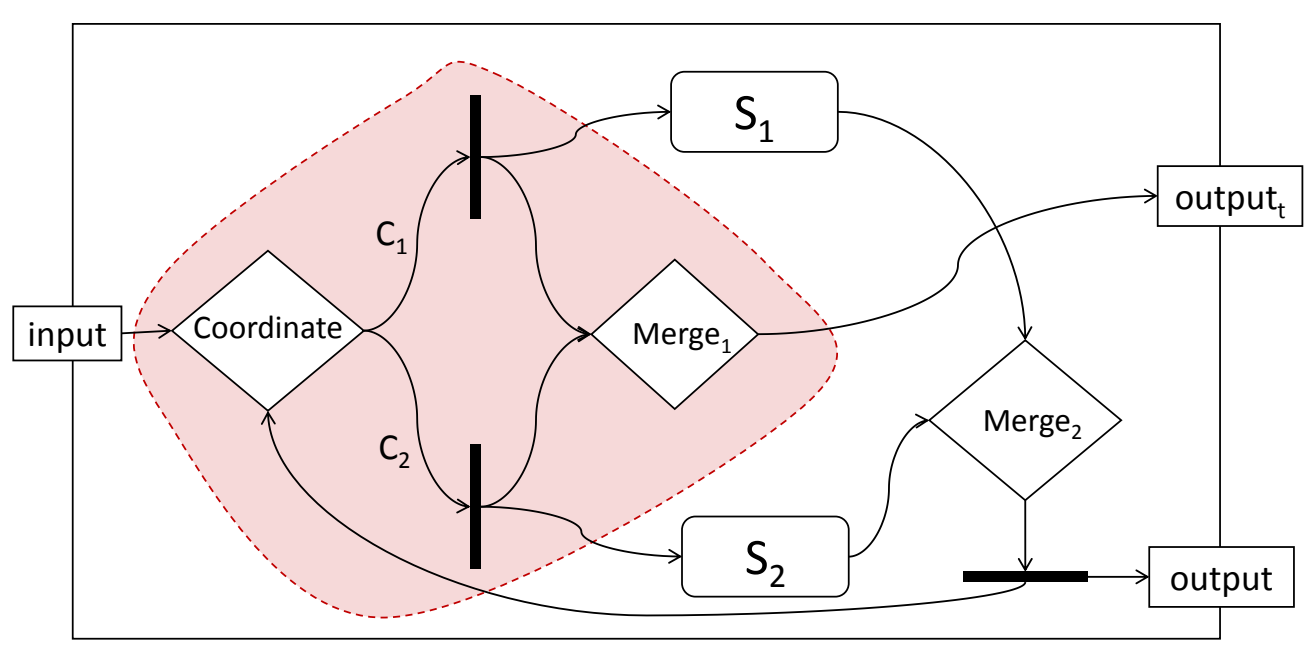

Figure 3: Activity of a multi-sever archetype architecture is devised using various activity constructs. $S_{1}$ and $S_{2}$ actions represent the the jobs service. $C_{1}$ and $C_{2}$ represent conditions for choosing flow directions. The nodes inside the dashed line area highlight the role of the activity control elements in the manipulation of the I/O flow.

in indicates arrival of a new job. The coordinator fetches the job and figures out its service time. It iterates through the secondary state variable that maintains the availability status of servers. If there is an available server, the job gets assigned to it and a transition to sending state is made. If there is no available server then the server remains at passive state and the job gets stored in FIFO queue. The inputs through port notify indicates a completion of job servicing and consequently the availability of the designated server. Therefore, a transition to sending is made if the queue has some jobs waiting to be served. The job is removed from the queue and dispatched to the available server. If there is no waiting job in the queue, then no transition is made and the model stays at the passive state.

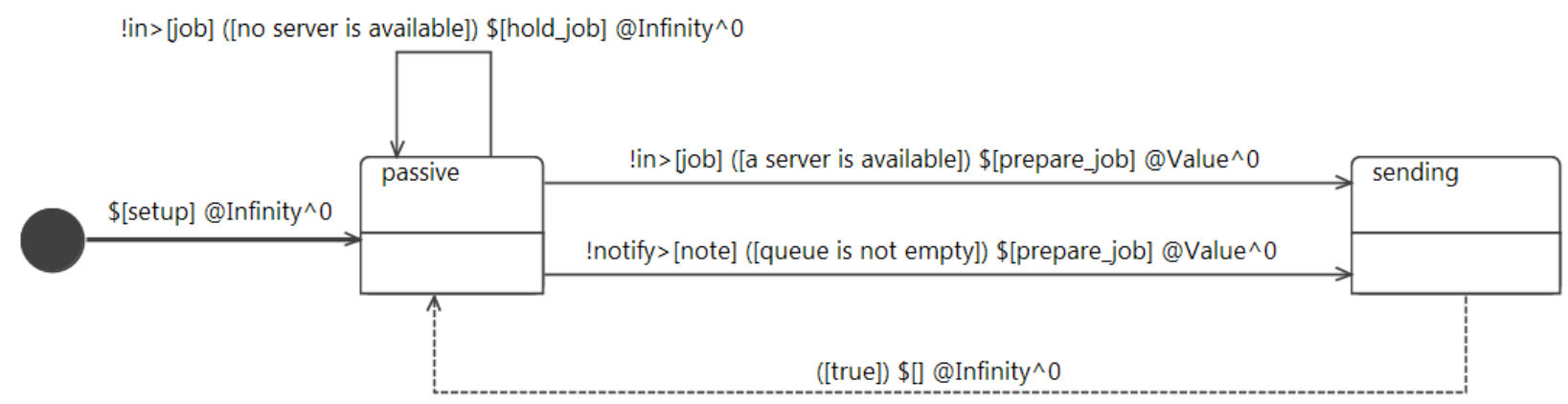

Figure 4: Modeling the coordinator statecharts in CoSMoS.

Both activities and statecharts are approached from a complementary standpoint. Together they provide a richer basis to specify behaviors although challenges may arise to establish a total perspective by which the notion of action and state are both fully considered. Neither alone can dictate the overall process of specifying behavior nor eliminate the necessity of manipulating codes to provide proper implementations.

\subsection{Constructing hierarchy within activities}

Based on the DEVS hierarchical model specification, an activity model can be conceptualized as a layer in a hierarchy. This leads an activity model to have higher abstractions using activity model elements. For 
example, a control node has a higher abstraction in relation to the primitive fork node. Higher level elements represent higher level concepts as compared with primitive elements. Thus, activity models at multiple abstraction levels can be constructed. In other words, higher level activity models can place constraints on the primitive elements used in activity models. Given the lack of hierarchy concept directly in the standard UML activity modeling, we consider using the activity model elements according to the DEVS model hierarchy and its corresponding abstract simulator. Constructing and coupling activity models for the atomic DEVS models leads to specifying coupled DEVS activity models which conform to the DEVS closure under coupling property. Behaviors of certain activity elements can be determined using their DEVS specifications. For example, a part of an external transition function can be defined using a fork node and a part of an output function can be defined as a decision node. Together, these activity elements can define an internal coupling between two atomic models contained in a coupled model. This approach supports defining hierarchical (higher-level) behavior at finer-grain abstraction levels. This is useful as behavior specification for hierarchical components can have additional details.

The model in Figure 3 has a flat structure (i.e., there is one coupled model which contains several atomic models). The hierarchy level for this model is one. Now, we redefine this model by replacing both of its atomic models that correspond to the servers $S_{1}$ and $S_{2}$ with two coupled models. In this hierarchical example, the model hierarchy level is limited to two. The contained coupled model which is created to correspond to the second server does not contain any coupled models. This example shows the communication semantics between the activity models according to the DEVS formalism as well as the semantics of the activity model elements. The aim is to help better understand and specify behaviors for coupled models using activity modeling. The DEVS simulation protocol provides the semantics for executing activities within atomic models as well as the input and the output communications between models. Communication between two activities belonging to different models are defined between any main activity and its nodes.

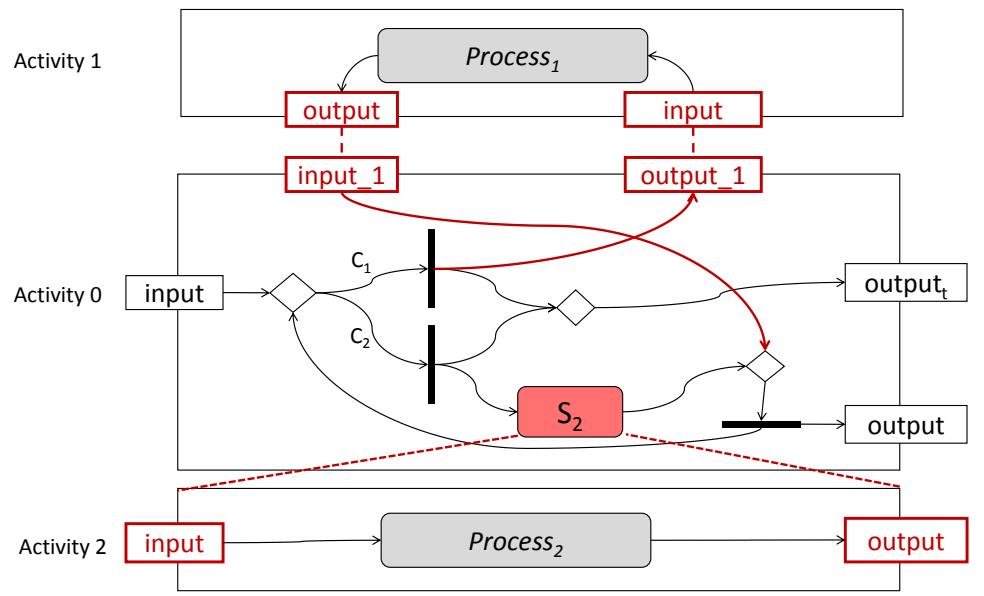

Figure 5: Hierarchical construction with activities.

The activity node $S_{1}$ is reconsidered in Figure 5 and replaced with a distinct activity (Activity 1 ) that is being communicated with through signals sent and received from the main Activity 0 . The node $S_{2}$ becomes also an activity (Activity 2), but it is contained within Activity 0. Elements in red (gray) color represents the model elements added to satisfy DEVS modularity. These two cases have different representations and hierarchy specifications in DEVS. The former leads to a coupled model at a hierarchical level corresponding to the main activity. The coupled models communicate through ports and external I/O couplings. The latter leads to establishing a new hierarchical level with the coupled model at hierarchy level one contained within the coupled model at hierarchy level two. Figure 5 illustrates how the two different cases can be presented in correspondence to the main activity. 
Alshareef and Sarjoughian

\section{RELATED WORK}

Enriching models at the higher levels of abstractions while providing supports for implementations via different code generation facilities has been of interest for many research efforts (e.g., (Lei et al. 2009, Cetinkaya et al. 2012, Sarjoughian and Markid 2012, Kapos et al. 2014, Mittal and Martín 2013, Sarjoughian et al. 2015)). These works address the problem holistically and yet thoroughly for the specifications in general (Lei et al. 2009, Cetinkaya et al. 2012, Mittal and Martín 2013, Kapos et al. 2014) and for behavioral specifications particularly (Sarjoughian, Alshareef, and Lei 2015). In (Lei et al. 2009) MDA is serving the aim of model transformation and primarily from structural vantage point. In (Cetinkaya, Verbraeck, and Seck 2012), structural metamodeling is the focus. In (Mittal and Martín 2013), the authors attempt to integrate the concepts in Model-Based System Engineering (MBSE) and Model-Driven Engineering (MDE) for the DEVS by employing modeling frameworks and tools that are based on MDA. The notion of activities is not used explicitly. In (Kapos et al. 2014), the integration is aimed at the System Modeling Language (SysML) based on the similarities between DEVS and SysML. Therefore, SysML definitions are closely examined in relation to DEVS concepts. A subset of SysML is aligned with DEVS by conforming to the SysML profile devised in close consideration to DEVS concepts. Models of such characteristics in the devised profile are made simulatable in a DEVS environment via transformation and code generation. In (Sarjoughian, Alshareef, and Lei 2015), the extension mechanism is accomplished by defining behavioral constructs and then incorporating them into the DEVS metamodel itself. It defines the action in the metamodel and associates it with different state transitions through meta-behavior based on EMF-DEVS (Sarjoughian and Markid 2012). The DEVS metamodel extends the Ecore metamodel of the Eclipse Modeling Framework (EMF). In (Kapos et al. 2014), activities of SysML are used to define parts of the atomic model behavior along with other state and parameter diagrams. The work targets SysML at large including its various metamodels such as those of block, state, parameter, constraint, and activity. The total SysML model is made simulatable after fully providing the specification required by a DEVS simulator. All the mentioned works comply to varying degrees with the MDA hierarchy to support creation of models that conform to their corresponding metamodels in a disciplined incremental manner.

This work focuses on notions of activities, mainly control and action. We propose an explicit definition of action and control in the DEVS formalism across different modeling layers. We also examine a relation where on one end is the hierarchical component models and on the other end resides action and control as fundamental units for identifying behaviors. As a result, richer activity models can be obtained. The notions of component and state are both used according to their underlying system-theoretic concepts. The action is introduced based on its definition such as in the UML (OMG 2012) and with consideration of existing DEVS variants that explicitly defines it such as ALRT-DEVS (Sarjoughian and Gholami 2015). These definitions are aligned across meta-layers and their corresponding implementations in CoSMoS are examined.

In (Harel and Politi 1998), the authors implement a system with different capabilities among which the ability to carry out simulation in one-step or in an interactive manner. The actions are defined to be carried out only instantaneously during a transition between different states. The formalism is defined for system under development (SUD) to provide a means for such a system to be developed by multiple modelers from different standpoints. The activity and actions are governed by the statecharts. In addition, component is defined for the physical module. In the DEVS formalism, the time advance function is defined for the atomic model. It could be assigned zero in the case of zero time advance; however, successive zero time state transitions must be finite in order to guarantee model legitimacy. Starting in the 1990s a variety of efforts began on using DEVS and various kinds of state machines as complementary paradigms, but they did not consider activity modeling.

In (Störrle, Hausmann, and Paderborn 2005), the authors consider Petri nets for ascribing semantics to activities in UML 2.0. The work considers the basic Petri nets formalism along with several extensions of it to address further some more expressive properties in activities such as control and data flow. Authors 
use extensions such as Colored Petri nets, Procedural Petri nets, and extension of the Procedural Petri nets. These extensions are used to try to incur further expressiveness encountered in activities but not in Petri nets. They conclude that mapping activities to Petri nets does not scale, meaning that extending the mapping with relatively non-trivial behavioral aspects thereby breaks the basic intuitions. Although limited, such transformations can lead to verification of certain properties in the context of the component-based model.

\section{CONCLUSION}

Some capabilities such as parallel processing and synchronization are exploited in the proposed modeling approach with the exemplar dual-server model. This has been made possible using the underlying DEVS formalism and defining models in correspondence with activity-based behavior specification. Such models can aid modelers to confine and develop simulations based on their well-defined semantics, both structurally and behaviorally. The proposed approach suggests the use of more intuitive diagrams to facilitate the development of models having complex dynamics. Hence, some of the described capabilities are not obvious due to using either the semi-formal or formal modeling methods.

The use of multiple abstractions exhibits the difficulty of handling ambiguities in some prominent modeling languages and terminologies. Rough definitions may be added, but they may increase the burden placed on the shoulders of modelers especially when the aim is to arrive at more useful simulations. Accounting for different abstractions can strengthen the process of incremental model development. Modelers are obliged to raise fundamental questions about their models starting from basic concepts. As a result, the discovery of such models and their corresponding suitable abstractions (MDA four-layer architecture) is guided, but remain in part unrestricted. Correctness of multiple entities in a given modeling framework is employed to increase the rigor needed for systems that continue to grow in both scale and complexity.

\section{ACKNOWLEDGEMENT}

We are thankful to the anonymous referees whose reviews helped us improve the presentation of our paper.

\section{REFERENCES}

ACIMS 2017. "CoSMoS”. Available at https://acims.asu.edu/software/cosmos/, version. 3.0.0.

ACIMS 2019. "DEVS-Suite". Available at https://acims.asu.edu/software/devs-suite/, version. 5.0.0.

Alshareef, A., and H. S. Sarjoughian. 2018. "Parallelism semantics in modeling activities". In Proceedings of the Symposium on Theory of Modeling and Simulation-DEVS Integrative M\&S Symposium, Spring Simulation Multi-Conference, Baltimore, MD, USA.

Alshareef, A., H. S. Sarjoughian, and B. Zarrin. 2016. "An approach for activity-based DEVS model specification". In Proceedings of the Symposium on Theory of Modeling and Simulation-DEVS Integrative M\&S Symposium, Spring Simulation Multi-conference, Pasadena, CA, USA.

Cetinkaya, D., A. Verbraeck, and M. D. Seck. 2012. "Model transformation from BPMN to DEVS in the MDD4MS framework". In Proceedings of the Symposium on Theory of Modeling and Simulation-DEVS Integrative M\&S Symposium, Spring Simulation Multi-conference, Orlando, FL, USA, pp. 28.

Fard, M. D., and H. S. Sarjoughian. 2015. "Visual and persistence behavior modeling for DEVS in CoSMoS". In Proceedings of the Symposium on Theory of Modeling \& Simulation-DEVS Integrative M\&S Symposium, Spring Simulation Multi-Conference, Alexandria, VA, USA, pp. 227-234.

Harel, D., and M. Politi. 1998. Modeling reactive systems with statecharts: the STATEMATE approach. McGraw-Hill, Inc. 
Henderson-Sellers, B. 2012. On the mathematics of modelling, metamodelling, ontologies and modelling languages. Springer Science \& Business Media.

Kapos, G.-D., V. Dalakas, M. Nikolaidou, and D. Anagnostopoulos. 2014. "An integrated framework for automated simulation of SysML models using DEVS”. Simulation vol. 90 (6), pp. 717-744.

Lei, Y., W. Wang, Q. Li, and Y. Zhu. 2009. "A transformation model from DEVS to SMP2 based on MDA". Simulation Modelling Practice and Theory vol. 17 (10), pp. 1690-1709.

Mittal, S., and J. L. R. Martín. 2013. "Model-driven systems engineering for netcentric system of systems with DEVS unified process". In Proceedings of the 2013 Winter Simulation Conference (WSC), pp. 1140-1151. IEEE Press.

OMG 2012. "Unified Modeling Language version 2.5.1”. https://www.omg.org/spec/UML/2.5.1/.

OMG 2017. "Semantics of a Foundational Subset for Executable UML Models (fUML) version 1.3". https: //www.omg.org/spec/FUML/1.3.

Sarjoughian, H. S. 2017. "Restraining Complexity and Scale Traits for Component-based Simulation Models". In Proceedings of the 2017 Winter Simulation Conference, Las Vegas, NV, USA. IEEE Press.

Sarjoughian, H. S., A. Alshareef, and Y. Lei. 2015. "Behavioral DEVS metamodeling". In Proceedings of the 2015 Winter Simulation Conference, Huntington Beach, CA, USA, pp. 2788-2799. IEEE Press.

Sarjoughian, H. S., and S. Gholami. 2015. "Action-level real-time DEVS modeling and simulation". Simulation vol. 91 (10), pp. 869-887.

Sarjoughian, H. S., and A. M. Markid. 2012. "EMF-DEVS modeling". In Proceedings of the Symposium on Theory of Modeling \& Simulation-DEVS Integrative M\&S Symposium, Spring Simulation MultiConference, Orlando, FL, USA, pp. 19.

Shoham, Y. 1989. “Time for Action”. In Proceedings of IJCAI, Volume 89.

Soley, R. et al. 2000. "Model driven architecture". OMG white paper vol. 308 (308), pp. 5.

Störrle, H., J. H. Hausmann, and U. Paderborn. 2005. "Towards a formal semantics of UML 2.0 activities". In In Proceedings German Software Engineering Conference, volume P-64 of LNI, pp. 117-128.

Wymore, A. W. 1993. Model-based systems engineering, Volume 3. CRC press.

Zeigler, B. P., A. Muzy, and E. Kofman. 2018. Theory of modeling and simulation: discrete event and iterative system computational foundations. Third ed. Academic press.

Zeigler, B. P., and H. S. Sarjoughian. 2017. Guide to Modeling and Simulation of Systems of Systems. Second ed. Springer.

\section{AUTHOR BIOGRAPHIES}

ABDURRAHMAN ALSHAREEF is a Ph.D. Candidate in Computer Science program in the School of Computing, Informatics, and Decision Systems Engineering (CIDSE) at Arizona State University. He can be contacted at alshareef@asu.edu.

HESSAM SARJOUGHIAN is an Associate Professor of Computer Science and Computer Engineering in the School of Computing, Informatics, and Decision Systems Engineering (CIDSE) at Arizona State University (ASU), Tempe, AZ, and the co-director of the Arizona Center for Integrative Modeling \& Simulation (ACIMS). He can be contacted at sarjoughian@asu.edu. 